\title{
Denture Esthetics: There is more than teeth replacement
}

\author{
Prateek Mishra ${ }^{1}$, Sneha S. Mantri ${ }^{2}$, Suryakant Deogade ${ }^{3}$, Pushkar Gupta ${ }^{4}$ \\ ${ }^{I}$ Post Graduate Student Department Of Prosthodontics, Hitkarini dental college and hospital, Jabalpur (M.P), \\ INDIA \\ ${ }_{2}^{2}$ Professor Department Of Prosthodontics, Hitkarini dental college and hospital, Jabalpur (M.P), INDIA \\ ${ }^{3}$ Professor Department Of Prosthodontics, Hitkarini dental college and hospital, Jabalpur (M.P), INDIA \\ ${ }^{4}$ Reader Department Of Prosthodontics, Hitkarini dental college and hospital, Jabalpur (M.P), INDIA
}

\begin{abstract}
The success of prosthetic treatment is predicted not only on the dentist's manual dexterity but also on the ability to understand the patient needs. Patient with complete edentulism undergoes many esthetic consequences among which sunken appearance of cheek has a great psychological effect on the individual that can add ages to a patient age. Prosthetic rehabilitation of such patient not only confines to replacement of missing teeth but also restoring the lost cheek support. This article focuses on simple, effective and a noninvasive method for restoring the slumped facial musculature by incorporating cheek plumper in the dentures and an innovative technique for the fabrication of detachable cheek plumper through custom made attachment.
\end{abstract}

Keywords: Denture, Esthetics, Cheek plumper

\section{Introduction}

The demand for age defying restorations has never been so prevalent in prosthodontics than it is right now. In current scenario, facial esthetics has became an integral part of complete denture prosthetic treatment and no longer confines to just replacement of missing teeth. The natural dentition or dentures provides support to the facial musculature which is responsible for the external form of the lips and cheeks. If the lips and cheeks are unsupported, muscles become weak and do not function properly which leads to wrinkling of skin and sagging of lips and cheeks that can add years to a person's age and hence have a detrimental psychological effect on the patient professional and social life. ${ }^{1}$

At times denture flange do provide support to the circum-oral muscles but fails to mimic the fullness of the cheeks. Cheek plumper, also known as the cheek lifting appliance is basically prosthesis for supporting and lifting the cheek to provide required support and esthetic that will increase the self-esteem of the patient. It is cited in literature for providing support to the cheek wherever and whenever deficient. ${ }^{2,3,4}$ This prosthesis is basically for supporting and plumping the cheek to provide a youthful appearance. A cheek plumper can be of two types: detachable and undetachable. It has been seen that undetachable cheek plumper have some limitations like increased weight which could hamper retention of the maxillary complete denture and makes it difficult to insert. Moreover it cannot be used in patients with limited mouth opening ${ }^{5}$.

To overcome the demerits of conventional undetachable cheek plumper, detachable check plumper has been tried that has proven more beneficial. In a detachable plumper prosthesis, plumper part can be detached from the complete denture. This clinical report illustrates the use of detachable cheek plumper with customized attachments which is connected with complete denture prosthesis using soft liner in a completely edentulous patient with hollow cheeks.

\section{Case Report}

A 51 year old male patient reported to the Department of Prosthodontics in Hitkarini dental college and hospital, Jabalpur with the chief complaint of missing teeth. On examination patient had completely edentulous upper and lower arches. Patient had lost his teeth over a period of 2 years as they were periodontally compromised and was edentulous for past 1 years. Apart from the intraoral findings, one of the major findings on extra oral examination was slumped cheeks.(Fig.1) Patient desired a prosthesis which would make his face look fuller and healthier. Treatment plan was formulated, keeping patient's demand in mind. It was decided to give patient complete dentures with detachable cheek plumper for the maxillary denture. Maxillary and mandibular impressions were made using impression compound (DPI PINNACLE, The Bombay Burmah Trading Corporation, Mumbai). Custom trays were made using autopolymerising acrylic resin (DPI-RR Cold Cure, DPI, Mumbai, India). Border molding was done using low fusing impression compound (Pinnacle Tracing sticks, DPI, Mumbai, India) and wash impressions were made with zinc oxide eugenol paste (Impression Paste, DPI, Mumbai, India). Jaw relations were recorded. For the try in appointment waxed denture were first tried for occlusion and esthetics. Wax template for cheek plumper were attached to the maxillary denture in the 
distobuccal region and were adjusted accordingly to give patient a more fuller appearance. After taking patient consent ,the waxed plumper was separated from the trial denture. Customised attachments were fabricated by molding modeling wax into two balls of $3 \mathrm{~mm}$ diameter attached to the base of plumper.

Acrylisation of dentures and check plumper were done in conventional way. (Fig.2) The finished and polished dentures were tried in the mouth. To get the snap fit between maxillary denture and check plumper, concavities were made in the denture which corresponded to the attachments in the cheek plumper and loaded with soft liner (PermaSoft Soft Denture Liner, Dentsply Austenal, York, Pa) of firm consistency by mixing powder liquid in the ratio of 3:1 .Fig.3)The cheek plumper were approximated back on the denture and held together for 10 minutes till complete polymerization. The resiliency of the soft liner allowed close approximation of the plumper with the denture and facilitated removal from the denture when required. (Fig.4) Patient was instructed on the use of plumpers and dentures were delivered after evaluating them for fit and esthetics. Recall appointment were scheduled after 1 day, 1 month and every 6 months.(Fig.5 and Fig.6)

\section{Discussion}

Denture esthetics have advanced ahead than mere selection of teeth on the factors of form, shape, color, arrangement and sex, it is more of harmonization of artificial with natural. ${ }^{6,7}$ The conventional cheek plumper has the major problem of retention and stability of maxillary denture due increased size and weight of the denture. It can also lead to muscle fatigue due to continuous use. In the present case detachable plumper prosthesis were planned to reduce weight of the final prosthesis and to allow ease in placement of the prosthesis. Detachable plumpers enabled the patient to remove the plumpers and use the denture if required. In the past, magnet retained plumper prosthesis have been used but they exhibit poor corrosion resistance and loss of magnetic property over a period of time. ${ }^{9,10}$ To overcome these disadvantages soft liner has been used. The resiliency of the soft liner allowed close approximation of the plumper with the denture and facilitated removal from the denture when required. However there are certain limitations associated with the soft liner like loss of resiliency over a period of time that requires its replacement over a period of 6-8 months. But the esthetic improvement and comfort delivered boosts the self esteem of patient far exceeds the disadvantage of the technique.

\section{Conclusion}

The dentist's ability to understand and recognize the problems of edentulous patients, to select the proper course of required treatment and reassure them has proven to be greatest clinical value.

By giving the patient detachable cheek plumpers with custom attachment the author has made an attempt to restore cheek fullness to the extent that comfort and function would permit and boost the self-esteem of the patient by improving his appearance.

\section{References:}

[1]. Bains JW, Elia JP. The role of facial skeletal augmentation and dental restoration in facial rejuvenation. Aesthet Plast Surg 1994;18:243-246.

[2]. Lazzari JB. Intraoral splint for support of lips in Bells palsy. J Prosthet Dent 1955; 5(4):579-581.

[3]. Larzen SJ, Cartern JF, Abrahamian. Prosthetic support for unilateral facial paralysis. J Prosthet Dent 1976; 35(2):192-201.

[4]. Hitoshi M, Chiaki K, Takashi O, Hisashi T. Lip plumper prosthesis for a patient with a marginal mandibulectomy: a clinical report. J Prosthet Dent 2004; 92(1):23-26.

[5]. Navitha Verma, Vidya Chitre, Meena Aras. Enhancing appearance in complete dentures using magnet retained cheek plumpers. J Indian Prostho Soc 2004; 4:35-38.

[6]. Clairf, Picard Jr. Complete denture esthetics. J Prosthet Dent 2005; 93:386-94.

[7]. Waliszewski M. Restoring dentate appearance: A literature review for modern complete denture esthetics. J Prosthet Dent 2005; 93:386-94

[8]. Tautin F. S. Denture esthetics is more than teeth selection. J Prosthet Dent 1978; 40:127-30.

[9]. Riley MA, Walmsley AD, Harris IR. Magnets in prosthetic dentistry. Journal of Prosthet Dent 2001;86(2):137-42.

[10]. Riley M, Williams A, Speight J, Walmsley A, Harris I. Investigations into the failure of dental magnets. The International journal of prosthodontics 1999;12(3):249. 


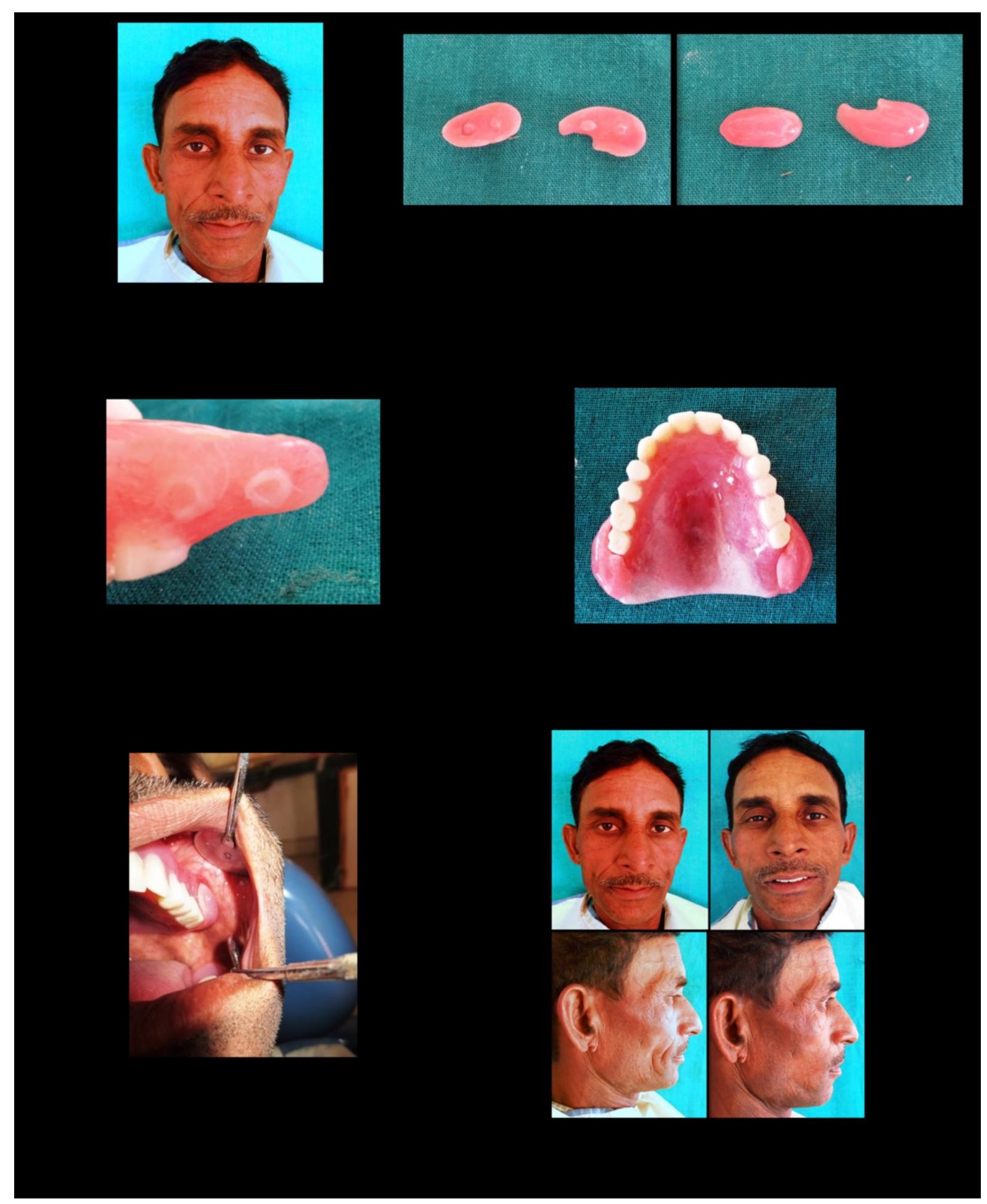

